\title{
Superhydrophobic hierarchical fiber/bead composite membranes towards efficient burns treatment
}

Weichang Li ${ }^{\text {a,b,\#} \text {, Qianqian } \mathrm{Yu}^{\mathrm{a}, \#} \text {, Hang Yao }}{ }^{\mathrm{c}, \mathrm{d}}$, Yue Zhu ${ }^{\mathrm{a}}$, Paul D. Topham ${ }^{\mathrm{e}}$, Kan Yue $^{\mathrm{a}, \mathrm{b}}$, Li Ren $^{\mathrm{d}, *}$, and Linge Wang ${ }^{\mathrm{a}, \mathrm{b}, *}$

${ }^{a}$ South China Advanced Institute for Soft Matter Science and Technology, School of Molecular Science and Engineering, South China University of Technology, Guangzhou 510640, China.

${ }^{\mathrm{b}}$ State Key Laboratory of Luminescent Materials and Devices, South China University of Technology, Guangzhou 510640, China

${ }^{c}$ School of Chemistry and Chemical Engineering, Yangzhou University, Yangzhou 225009, China

d National Engineering Research Center for Tissue Restoration and Reconstruction, Guangzhou 510006, China

${ }^{\mathrm{e}}$ Aston Institute of Materials Research, Aston University, Birmingham, B4 7ET, UK

\section{Corresponding Authors}

\# W. Li and Q. Yu contributed equally to this work.

L. Ren, Email: psliren@scut.edu.cn, Tel: +86-20-87114645

L. Wang, Email: lingewang@scut.edu.cn, Tel: +86-20-22237367

\begin{abstract}
One of the current challenges in burn wound care is the development of multifunctional dressings that can protect the wound from bacteria or organisms and promote skin regeneration and tissue reconstitution. To this end, we report the design and fabrication of a composite electrospun membrane, comprised of electrospun polylactide: poly(vinyl pyrrolidone)/polylactide:poly(ethylene glycol) (PLA:PVP/PLA:PEG)
\end{abstract}


core/shell fibers loaded with bioactive agents, as a functionally integrated wound dressing for efficient burns treatment. Different mass ratios of PLA:PVP in the shell were screened to optimize mechanical, physicochemical, and biological properties, in addition to controlled release profiles of loaded antimicrobial peptides (AMPs) from the fibers for desirable antibacterial activity. Fibroblasts were shown to readily adhere and proliferate when cultured on the membrane, indicating good in vitro cytocompatibility. The introduction of PLA beads by electrospraying on one side of the membrane resulted in biomimetic micro-nanostructures similar to those of lotus leaves. This designer structure rendered the composite membranes with superhydrophobic property to inhibit the adhesion/spreading of exogenous bacteria and other microbes. The administration of the resulting composite fibrous membrane on burnt skin in an infected rat model led to faster healing than a conventional product (sterile silicone membrane) and control detailed herein. These composite fibrous membranes loaded with bioactive drugs provide an integrated strategy for promoting burn wound healing and skin regeneration.

Keywords: electrospinning, fibers, beads, micro-nanostructures, superhydrophobic, antibacterial property, burns healing 


\section{Introduction}

Burns constitute one of the worst forms of trauma and remain a global public health issue.[1,2] Medical treatment of full-thickness skin burns remains a major clinical challenge.[3, 4] Currently, autologous skin grafting is the 'gold standard' for burn wound repair. However, this procedure is often accompanied with high risks of secondary morbidity during graft harvesting, and the amount of available healthy skin is also limited.[5,6] Therefore, the application of artificial biomedical materials is widely considered as a promising alternative to autografts.[7] The repair and reconstruction of burnt skin follows a series of complex processes involving inflammation, cell proliferation and tissue regeneration, and are further complicated by invasion of infectious species, dehydration, and repeated additional trauma caused by surgery or wound cleaning.[8] To avoid these complications, an ideal wound dressing should possess specific key functions: 1) prevent invasion of microorganisms and reduce infection; 2) slow down dehydration and retain a favorable moist environment around the wound; and 3) mimic the structural and biological features of the natural extracellular matrix (ECM) to promote complete regeneration of injured skin tissue.[911]

In recent years, wound dressings prepared by electrospinning techniques have attracted increasing attention.[12] Since the similarity of electrospun fibers and ECM in many structural and properties, such fiber-based materials can provide support for cells, regulate cellular activities, and allow facile transportation of nutrients and waste.[12] The high specific surface area and porous nature of electrospun fibers create 
opportunities for controlled delivery of antibacterial drugs and other therapeutic agents,[12-14] which can potentially be programmed to regulate the complex healing mechanism. $[15,16]$ Furthermore, coaxial electrospinning is a technique that allows the fabrication of multicomponent core/shell fibers.[17] Since the compositions and properties of the inner and outer layers in core/shell fibers can be independently tuned, coaxial electrospinning provides a feasible strategy to achieve balanced performance between drug loading efficiency and drug release kinetics.[18, 19]

Wound dressings can also play a role in preventing the wound from invasion and contamination of exogenous bacteria and other microbes. To this end, the introduction of superhydrophobic properties to the exposed dressing surface would effectively prevent adhesion of microbes. Recently, superhydrophobic interface has been extensive studied for applications such as smart cell capture/release systems, self-cleaning coatings and anti-bioadhesion surfaces.[20-22] It is now well-documented that surface superhydrophobicity is dependent on both the chemical composition and the formation of hierarchical micro- and nanostructures (or surface roughness).[23] Bio-inspired surfaces with lotus leaf-like micro-nanostructures have been fabricated and studied for their superhydrophobic properties.[24, 25] Interestingly, similar rough surfaces can be feasibly achieved via electrospraying to deposit microbeads,[26-28] which can be combined with electrospinning to fabricate composite membrane materials with asymmetrically modified surface properties.

Herein, we report the fabrication of a composite fibrous membrane material composed of fiber/bead fibers as a fully integrated, multifunctional would dressing for effective 
burn treatment. Specifically, a mixture of poly(lactic acid):poly(vinyl pyrrolidone) (PLA:PVP) with antimicrobial peptides (AMPs) was used as the shell layer, while a mixture of PLA:poly(ethylene glycol) (PLA:PEG) blended with curcumin was used as the core. AMPs are short natural peptides that have a broad spectrum of antimicrobial activity, short contact time to induce killing, and low susceptibility to develop bacterial resistance.[29, 30] The motivation to make use of core/shell fibers from coaxial electrospinning is two-fold. Firstly, controlled release of AMPs from the shell layer can be used to deal with acute inflammatory responses in primary wound healing and, secondly, subsequent sustained release of curcumin from the inner core can accelerate wound healing and tissue reconstitution at the mid to late wound healing stages, due to the known anti-inflammatory and antioxidant activities of curcumin.[31] Furthermore, one surface of the electrospun membrane has been coated with PLA microbeads by electrospraying to impart superhydrophobicity and anti-bioadhesive properties, to effectively block the invasion of bacteria. The high antimicrobial activity of the resulting composite material was tested in vitro against Escherichia coli (E. coli) and Staphylococcus aureus (S. aureus). Finally, the capability of this dressing material in promoting wound healing in vivo has been evaluated using a full-thickness burn wound model in infected Sprague-Dawley (SD) rats. Our work unveils a promising strategy for the development of functionally integrated wound dressings for burn wound care.

\section{Materials and methods}

\subsection{Materials}


$\operatorname{PLA}\left(M_{n}=50,000 \mathrm{~g} / \mathrm{mol}\right)$ and PVP $\left(M_{n}=130,000 \mathrm{~g} / \mathrm{mol}\right)$ were purchased from Nature Works (LLC, US) and Aladdin Reagent (Co., Ltd., China), respectively. PEG $\left(M_{n}=\right.$ $10 \mathrm{~K} \mathrm{~g} / \mathrm{mol}$ with a melting temperature of $\left.63^{\circ} \mathrm{C}\right)$ was purchased from SCRC (Co., Ltd, China). Dichloromethane (DCM, General-Reagent) was obtained from Aladdin. Penicillin, fetal bovine serum (FBS), phosphate buffer solution (PBS) and streptomycin were provided by the Life Technologies (Grand Island, NY). NIH/3T3 cells were supplied by the Cell Center of Jinan University and maintained in specific medium at $37^{\circ} \mathrm{C}$ in an atmosphere containing $5 \% \mathrm{CO}_{2}$, used as an appropriate degree of confluence. Gram-negative bacteria (Escherichia coli. ATCC8739) and gram-positive bacteria (Staphylococcus aureus. ATCC1901) were purchased from Guangdong Microbiology Culture Center. Antimicrobial peptides HHC36 were purchased from GL Bio (GL Biochem, Shanghai, Ltd.).

\subsection{Electrospinning}

Shell solutions ( $6 \mathrm{wt} \%$ in total) were prepared by dissolving different ratios of PLA and PVP (5:5, 7:3, 8:2 and 9:1, w/w) in DCM. The core solution was prepared with PLA and PEG $(1: 1, \mathrm{w} / \mathrm{w})$ in DCM at a total concentration of $13 \mathrm{wt} \%$. Desired amounts of AMPs (HHC36) and curcumin were added to the shell and core solutions, respectively, and were mechanically stirred for $24 \mathrm{~h}$ at room temperature before use. For electrospinning, the solutions were injected via a metal coaxial nozzle at flow rates regulated by two syringe pumps (LSP01-1A, Baoding Longer Precision Pump Co. Ltd., China). And the inner and outer diameters of the coaxial nozzle were 0.3 and $1.0 \mathrm{~mm}$, respectively. Coaxial Electrospinning was operated at $20 \mathrm{kV}$ with a nozzle-to-collector 
distance of $15 \mathrm{~cm}$. During coaxial electrospinning, core/shell fibers composed of PLA:PEG composite fibers embedded in PLA:PVP composite fibers were formed and collected using a plate collector (as shown in Schematic 1). The obtained membranes were transferred to a vacuum oven to fully remove residual organic solvent. The chemical composition of electrospun fibers were identified by Fourier transform infrared (FT-IR) spectroscopy (Fig. S1 in ESI).
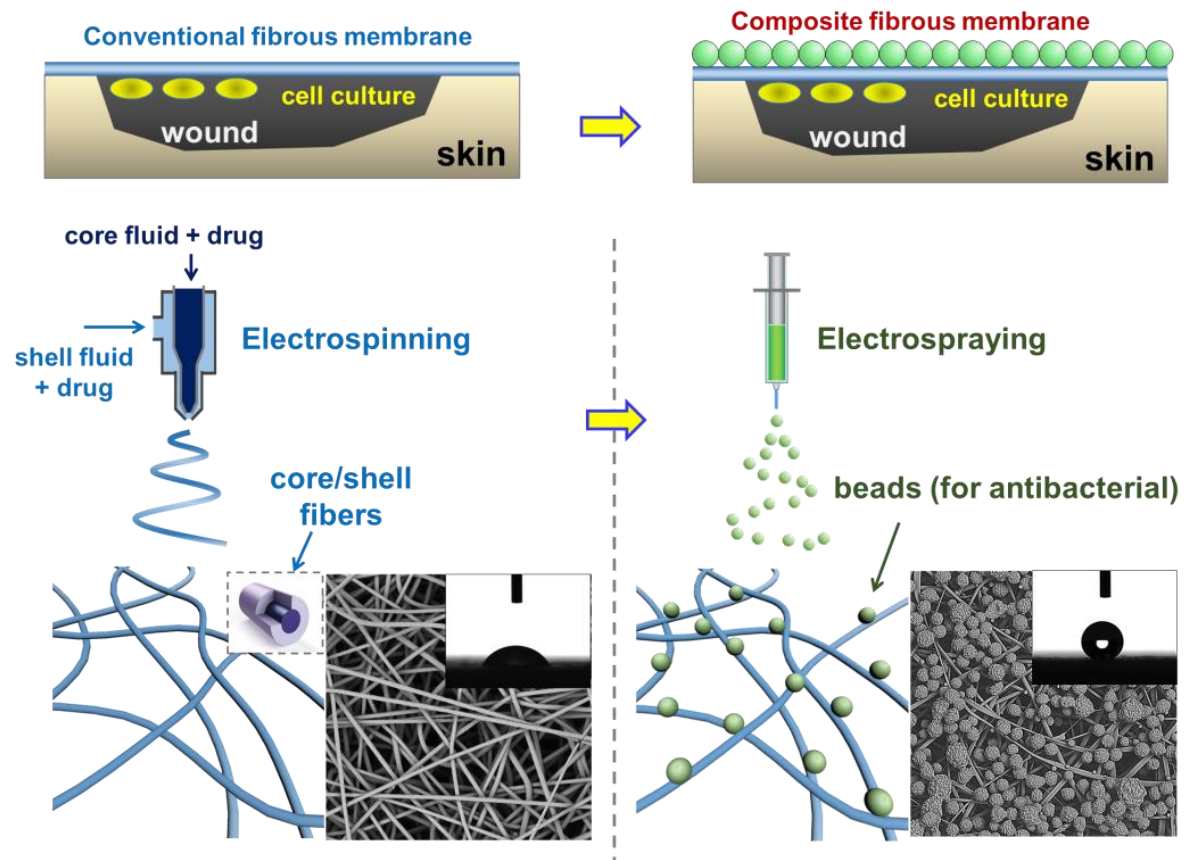

Schematic 1. Illustration of preparation process of core/shell fibers via coaxial electrospinning (left) and the membranes modified using beads via electrospraying (right).

To form hierarchical micro-nanostructured superhydrophobic coatings, PLA microbeads were deposited on the membrane by electrospraying a $5 \mathrm{wt} \%$ PLA solution in DCM. In this step, the fabricated fibrous membranes were used as the collector. Different volumes of PLA solution $(100,200,300,400$ or $500 \mu \mathrm{L})$ were consumed in this modification process to tune the morphology. The resulting composite membranes were similarly dried and characterized by FT-IR spectroscopy (Fig. S1). 


\subsection{Characterization}

Morphology of the electrospun fibrous membranes were characterized by scanning electron microscopy (SEM, Phenom Pro, Holland). Membrane samples were firstly plated withgold/palladium (Au/Pd) before the SEM examination. Core/shell structures of the fibers were confirmed using transmission electron microscopy (TEM, Hitachi h7650, Japan) by samples collected on carbon-coated copper grid. Image analysis was performed using ImageJ (public domain produced by Wayne Rasband from National Institute of Health) to ensure the average diameters (with standard deviations) of the fibers. Water contact angle (WCA) was measured by an OCA 20 contact-angle system (Dataphysics, Germany) equipped with a tilting base. Mechanical tests were determined by a tensile tester (BOSE ElectroForece3200, USA). At least 5 replicates of each membrane were tested.

\subsection{In Vitro Cell Culture}

NIH/3T3 cells were cultured in a DMEM media containing FBS (10\%), penicillin (100 $\mu \mathrm{g} / \mathrm{mL})$ and streptomycin $(100 \mu \mathrm{g} / \mathrm{mL})$ at $37{ }^{\circ} \mathrm{C}$ in an atmosphere containing $5 \% \mathrm{CO}_{2}$. The cells were passaged weekly with the culture media changed every other day. Before seeding, the cells were disposed by trypsin and re-suspended in culture medium.

For in vitro cytocompatibility tests, different membrane samples were sterilized under UV irradiation and then immersed in culture medium for $24 \mathrm{~h}$. The leaching liquor was used as growth medium for NIH/3T3 cells. Viability of the seeded NIH/3T3 was carried out with CCK-8 (Dojindo, Kumamoto, Japan) assay. Then the optical density (OD) of 
the solutions were recorded by a microplate reader (Varioskan Flash 3001, Thermo, Finland) at a wavelength of $450 \mathrm{~nm}$.

For in vitro cell culture on electrospun membranes, a representative fibrous membrane made of a PLA:PVP (w/w = 7:3) shell layer was selected. Membrane samples were preequilibrated with the culture media for $12 \mathrm{~h}$ at the same culture condition. NIH/3T3 was then seeded and cultured for 1 day or 3 days, after which the films were fixed with glutaraldehyde and rinsed with PBS, followed by dehydration with gradient ethanol and observation using SEM.

\subsection{In Vitro Release Profiles}

In vitro release curves of AMPs from the shell layer and curcumin from the core were determined using UV-vis spectroscopy (Shanghai UNICO, UV3802, China). Absorption peaks of AMPs and curcumin were filed to evaluate the amount of released medium. Membrane specimens were prepared in triplicate and immersed in PBS under the ambient temperature of $37{ }^{\circ} \mathrm{C}$. At the given time points, the PBS $(1 \mathrm{~mL})$ was extracted and measured to determine the release kinetics. Equivalent amounts of fresh PBS were added into the release solution to continue incubation. Release percentages were calculated based on the amount of initially loaded drug. [32]

\subsection{Antibacterial activity tests}

Antimicrobial experiments against both S. Aureus (gram-positive) and E. coli (gramnegative) were performed according to a previously described procedure. [33] Briefly, single colonies of S. aureus and E.coli were incubated with shaking at $155 \mathrm{rpm}$ in Luria- 
Bertani (LB) media overnight at $37{ }^{\circ} \mathrm{C}$. The cultured bacteria were diluted with fresh LB media and further cultured for $5 \mathrm{~h}$ with shaking at the same temperature. The bacteria with logarithmic-phase was re-suspended in PBS buffer with a concentration of $10^{7} \mathrm{CFU} / \mathrm{mL}$. Before seeding, all the membrane samples of different compositions were cut into round pieces of the same size, sterilized, and then placed in petri dishes. The bacterial suspension was placed onto per sample to completely overspread the surface.

After incubation, viability of cultured $S$. aureus and $E$. coli on membrane samples were tested by the Live/Dead assay of acridine orange (AO) and ethidium bromide (EB) fluorescent dyes.[34] Bacteria were collected by oscillating and centrifugation, washed with PBS three times, then incubated with the dye $(0.01 \mathrm{~mol} / \mathrm{mL})$ in PBS for $15 \mathrm{~min}$ in the dark at $25^{\circ} \mathrm{C}$. After raised, the stained bacteria were observed with a laser scanning confocal microscope (LSCM) (Leica TCS SP5 II, Leica Microsystems). For further evaluate the viability of bacteria, the diluted bacteria suspensions were also placed onto solid agar substrates. After incubation under the same conditions, the samples were rinsed with PBS and dehydrated with gradient ethanol for imaging using SEM.

\subsection{In Vivo Wound Healing Mode}

All rat studies were endorsed by the Jinan University Animal Procedures Committee. Sprague-Dawley (SD) rats (female, 6-week old, ca. $200 \mathrm{~g}$ ) were carrying out to appraise the in vivo burn wound repair performance of the membranes. Animals were anaesthetized by injecting pentobarbital sodium into the intraperitoneal and the back hair of each rat was shaved using an electric razor and a depilatory cream. The upper 
back skin was burned with a hot cylindrical metal $\left(90^{\circ} \mathrm{C}, 25 \mathrm{~s}\right)$ to create a full thickness defect of burn wound. Then, the burned tissue was clinically removed and infected wounds were created by treating with bacteria suspension (S. aureus and E. coli). There were four wounds were generated on the back skin of per rat. The wounds were covered with either sterile silicone membrane, fibrous membrane, composite fibrous membrane, or composite fibrous membrane loaded with drugs. During the healing process, no other medicine was applied, and the areas of the skin wounds were recorded. At day 3, 7, 10 and 14 post wounding, the rats were euthanized using $\mathrm{CO}_{2}$ gas, and specimens of the impaired skin were gathered for the histological analysis.

\subsection{Histological Analysis}

Tissue sections harvested from the rats of different group were fixed in $10 \%$ neutral buffered paraformaldehyde and embedded with paraffin, then sectioned to obtain the thick samples. Representative sectioned samples were stained with hematoxylin and eosin (H\&E, Sigma-Aldrich Co.) and Masson's trichrome (MT) for collagen fibers. The samples with stained were observed by optical light microscopy (Axio Scope A1 FL; Carl Zeiss, Wetzlar, Germany). All histological analyses were performed using at least three samples per group per time point and images presented are representative of all replicates.

\subsection{Immunohistochemistry}

General immunohistochemical staining method was applied, briefly, paraffinembedded sections were de-paraffinized, rinsed with PBS repeatedly, and then blocked 
with $5 \%$ serum for $0.5 \mathrm{~h}$. Sections were then stained with primary antibodies against interleukin-1 (IL-1, Abcam, Cambridge, UK), tumor necrosis factor-a (TNF- $\alpha$, Abcam Inc., Cambridge, MA, USA), or interleukin-6 (IL-6, Abcam, Cambridge, UK) overnight at $4{ }^{\circ} \mathrm{C}$. The sections were treated and incubated after washing by PBS at $37^{\circ} \mathrm{C}$ for 0.5 h. Subsequently, the sections were detected with 3,3-diaminobenzidine tetrahydrochloride (DAB) substrate (Invitrogen, Carlsbad, CA, USA) and counterstained with hematoxylin. The samples were detected using a digital pathology system (Aperio CS2, Leica, Germany).

\subsection{Statistical Analysis}

To the statistical inquiry, all statistical computations were performed by one-way ANOVA subsequently using Bonferroni's post-hoc test (GraphPad Prism 5.02) software. Data are expressed as the mean \pm standard deviation (SD) of assessment $(* p$ $<0.05)$.

\section{Results and discussion}

\subsection{Fabrication and characterization of core/shell fibrous membranes}

In recent years, the fabrication of core/shell fibers from coaxial electrospinning has been extensively studied.[35, 36] In this process, a multilayered, coaxially aligned nozzle is used to simultaneously conduct fluids of different compositions. In principle, the resulting composite fibers can offer a higher level of flexibility for rational design of materials with tailored mechanical properties and drug release profiles. Here, PLA has been selected as the matrix material for fibrous membranes, due to its excellent 
miscibility with other polymers, biocompatibility and biodegradability.[37, 38] Many blending systems based on PLA have been previously processed by electrospinning, including PLA:PEG,[39, 40] PLA:PVP,[37] PLA:poly(vinyl alcohol),[41] PLA:poly(E-caprolactone),[42, 43] and so on. To achieve different payload release profiles from the core/shell fibers, mixtures of PLA:PVP and PLA:PEG were designed as the shell and core compositions, respectively. In general, release kinetics depend primarily on the properties of the shell layer. Thus, the core composition has been fixed at PLA:PEG $=1: 1(\mathrm{w} / \mathrm{w})$, while the ratios of PLA:PVP were varied for the shell layer (PLA:PVP = 5:5, 7:3, 8:2, 9:1, w/w).

SEM images of electrospun fibers from these different core/shell combinations show similar randomly oriented, intertwined fibrous morphologies, with uniform fiber diameters (Fig. 1a-1d). The magnified SEM images (shown as insets) show that the fiber surfaces became rougher as the mass ratio of PLA to PVP increased from 5:5 to 9:1. As expected, such uneven surfaces full of etched and porous features can improve the release rate of drugs (vide infra). This morphological change is attributed to the decreased solution viscosity when the PVP content is low. The core/shell structure of these fibers was observed via TEM. As shown in Fig. 1e, the TEM image of a representative fiber (PLA:PVP $=7: 3$ in the shell) shows a clear boundary between the core and shell layers, indicating successful fabrication of core/shell structured electrospun fibers. 

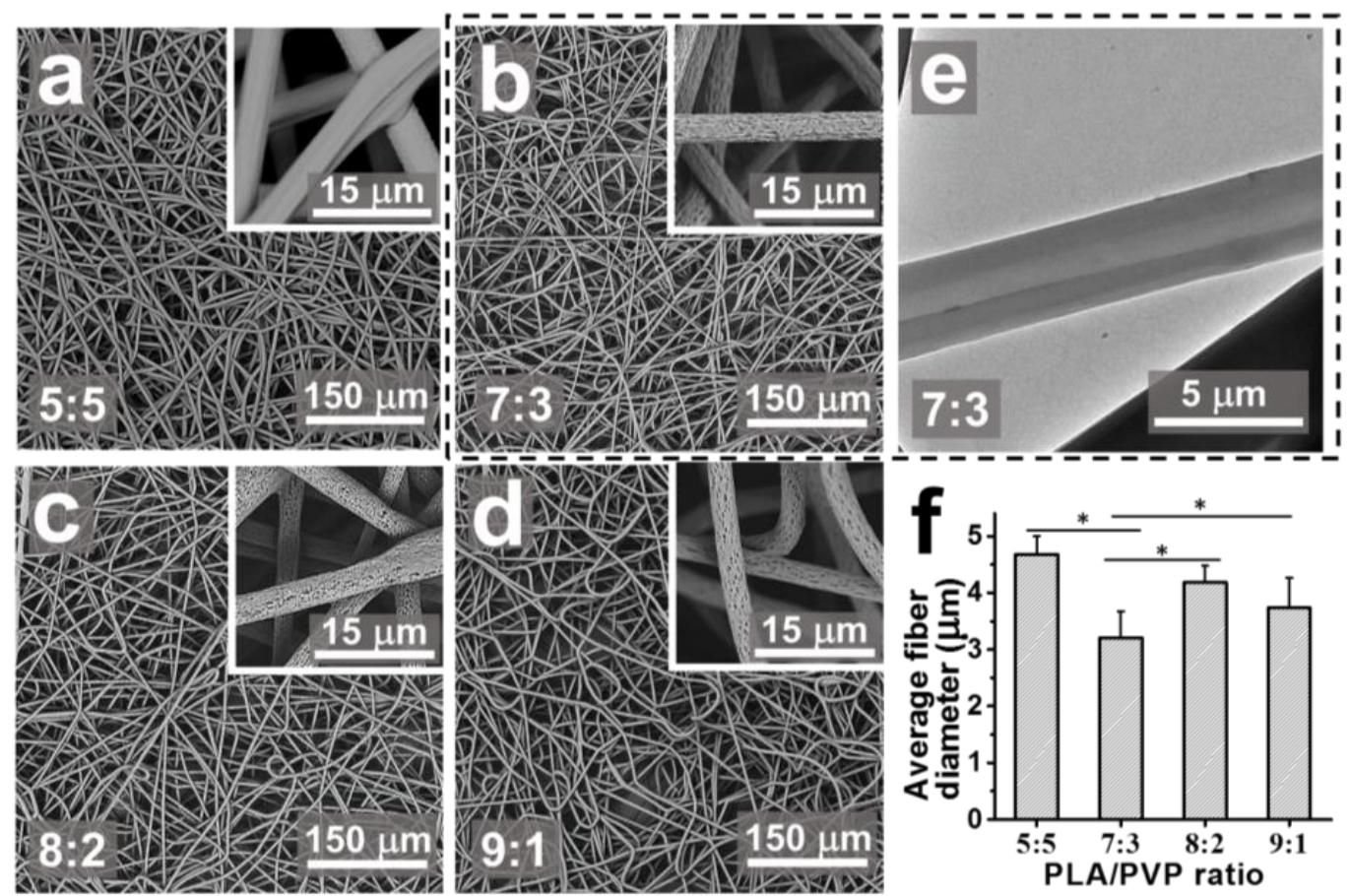

Fig. 1. SEM images of the fibrous membranes prepared with different PLA:PVP mixture ratios of shell solutions of 5:5 (a), 7:3 (b), 8:2 (c) and 9:1 (d). TEM image of core/shell structure obtained from the fiber of 7:3 (e). Fiber diameter distributions of the fibrous membranes (d).

The average fiber diameters obtained from different mass ratios of PLA/PVP in the shell solutions were $4.68 \pm 0.32 \mu \mathrm{m}, 3.21 \pm 0.47 \mu \mathrm{m}, 4.18 \pm 0.30 \mu \mathrm{m}$ and $3.73 \pm 0.53$ $\mu \mathrm{m}$ for 5:5, 7:3, 8:2 and 9:1 (PLA:PVP, w/w), respectively (Fig. 1f). Previous studies on polymer solution electrospinning suggest that decreasing the viscosity of the electrospinning solution generally decreases fiber diameters.[44] In a blending system, other factors, such as blending ratio and miscibility, can also affect the diameter of electrospun fibers.[45] Among the four different compositions of the shell solutions, the mass ratio of 7:3 (PLA:PVP, w/w) resulted in fibers with the smallest diameter, while the mass ratio of 5:5 led to the biggest diameter (Fig. 1f). 
Mechanical properties of electrospun core/shell fibers also depend on many factors, including fiber composition and experimental conditions for electrospinning.[46] The shell solution connects both the shell/air and shell/core interfaces and therefore plays a crucial role in determining the mechanical strengths of the resulting core/shell fibers. Our previous study on the reinforcing effect of fillers in electrospun fibers show that the mechanical strength can be optimized by controlling the mixing ratio, specifically depending on the nature of the matrix and the matrix-filler interactions. Herein, the mechanical properties of fibers obtained from different shell solutions (mass ratios of PLA:PVP = 5:5, 7:3, 8:2 and 9:1, w/w) have been studied in unidirectional tensile tests. From the characteristic stress-strain curves for different samples (Fig. 2a), it can be seen that mechanical properties of the fibers, including maximum tensile strength (Fig. 2b) and Young's moduli (Fig. 2c) depend on the shell layer compositions. The maximum tensile strain did not significantly change for all of the tested samples with different shell layers (Fig. 2d). Of all of the four ratios, 7:3 (PLA:PVP w/w) resulted in membranes with the highest tensile strength $(1.88 \pm 0.15 \mathrm{MPa})$ and Young's modulus $(9.76 \pm 1.49 \mathrm{MPa})$, thus suggesting the optimized shell layer composition in terms of mechanical performance. 

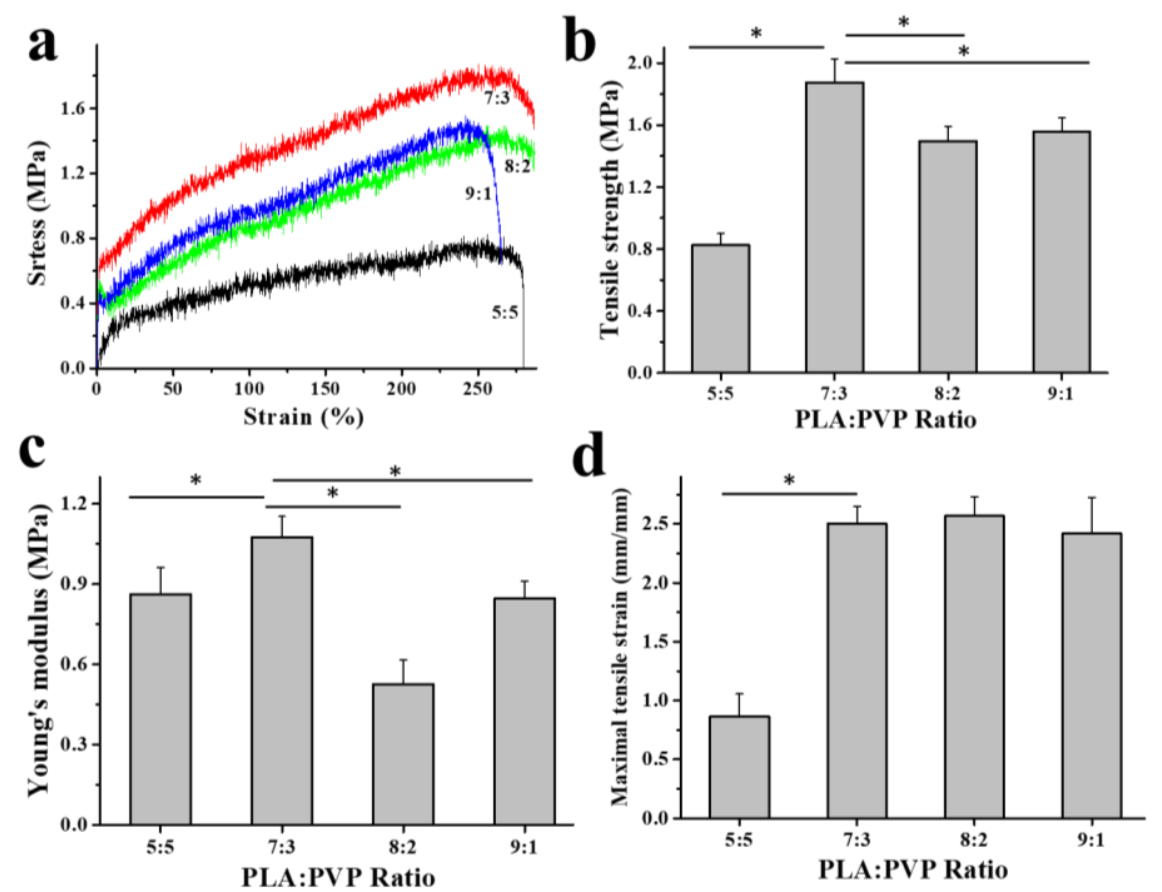

Fig. 2. Tensile stress-strain (a), tensile strength (b), Young's modulus (c) and maximal tensile strain (d) for fibrous membranes of different PLA:PVP mixture ratios of shell solutions.

The proliferation and differentiation of fibroblasts is an extremely essential factor during the wound healing. Cytocompatibility of the electrospun membranes were assessed using NIH/3T3 cells. The cells were first cultured with the membrane samples in vitro, and their viability was evaluated using the CCK8 assay, as shown in Fig. $3 \mathrm{a}$. All experimental groups showed increased OD values with increased culture time. No significant difference in OD value was observed for membranes with different shell layer compositions (mass ratios of PLA:PVP = 5:5, 7:3, 8:2 and 9:1, w/w), indicating that none of the membranes exhibited clear cytotoxicity. To further determine the interactions of NIH/3T3 cells with the membranes, the cells were seeded directly onto the membrane surfaces. Under SEM observations (Fig. 3b and 3c), cells seeded on a representative membrane (PLA:PVP $=7: 3$ ) at day 1 and day 3 showed elongated, 
spindle-like shapes, showing the capability of the membrane substrates to support cell adhesion and attachment. Furthermore, increased cell density was identified when comparing the image at day 3 (Fig. 3c) with that at day 1 (Fig. 3b). Therefore, the adhesion and proliferation of NIH/3T3 cells on these fibrous membranes demonstrated excellent in vitro cytocompatibility and favorable cell-membrane interactions.
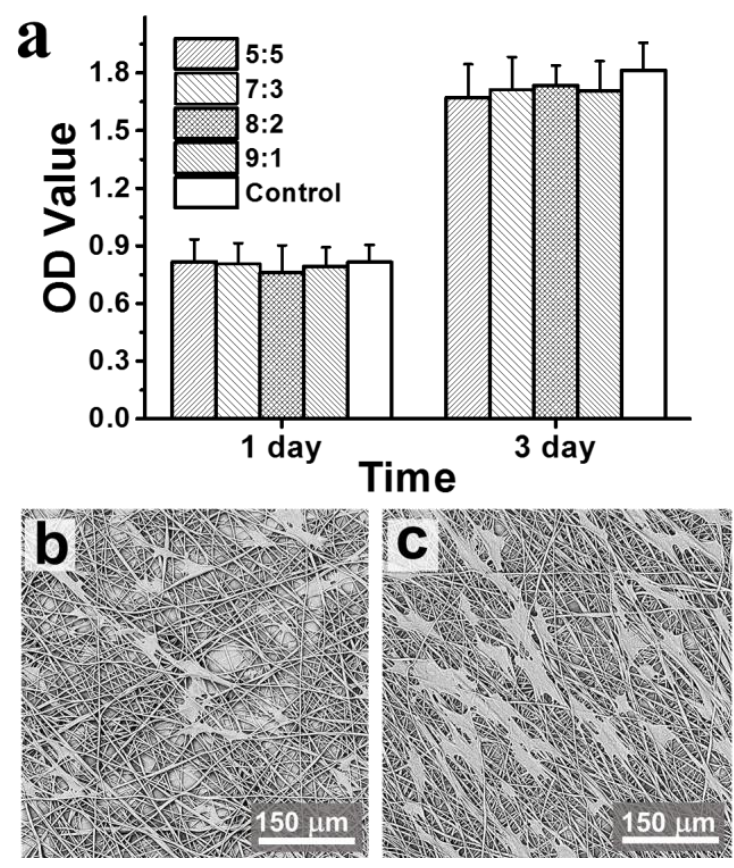

Fig. 3. Cell viability with different membranes as a function of culture time (a). Cell morphology on fibrous membrane of 7:3 cultured for 1day and 3 days.

For wound dressing applications, the membranes should ideally be able to keep a moist environment around the skin wound and absorb the exudates from the wound surface for a sustained period of time. In vitro swelling experiments (Fig. S2) show that membranes made of fibers with a shell layer composition of PLA:PVP = 7:3 (w/w) gave the highest water absorption capability. Moreover, the good mechanical properties and thermal stability (as measured by thermogravimetric analysis, TGA, Fig. S3) indicated that these membrane materials can be readily stitched and serve as stable 
wound dressings. In short, by combining all of the physical characterization data, the optimized shell layer composition was PLA:PVP $=7: 3(\mathrm{w} / \mathrm{w})$. Consequently, this composition was selected for the in vitro release and in vivo studies, as discussed in the following sections.

\subsection{In vitro drug release studies from the core/shell fibrous membranes}

The repair of burn wounds involves many simultaneous and cascading events, such as inflammation management, cytokine and growth factor secretion, temporary skin replacement, and tissue reconstitution. Therefore, an ideal wound dressing should provide a range of functions to aid the wound repairing process, including the controlled delivery of bioactive agents. One unique advantage of core/shell fiber-based dressing materials is the capability to disperse different bioactive agents into the core/shell layers via coaxial electrospinning, which can be explored to tailor the release profiles of disparate components from the resulting dressing substrates. This programmed drug release function can be designed to coincide with the multistep wound healing process to promote its completion.

In this study, AMPs were loaded in the shell layer and curcumin in the core layer to manage the acute bacterial infection and later-stage inflammation, respectively. As shown in Fig. 4, in vitro release of both payloads in core/shell fibers indicated initial burst release behavior during the first few hours, then a more sustained release phase within the next few days. Notably, the release of encapsulated AMPs in the shell layer was much faster than that of loaded curcumin in the core layer, as expected. The percentage of cumulative release of AMPs reached $c a$. $70 \%$ within $24 \mathrm{~h}$ and over $90 \%$ 
within $72 \mathrm{~h}$, while the release of curcumin could be extended to around 5 days (Fig. 4). Such different drug release rates from different layers of core/shell fibers can be explained by simple diffusion-controlled dissolution models, $[47,48]$ in which the shell layer acts as a protection for the payload in the core. Such different release profiles show clear potential to meet the requirements to diminish bacterial infection and manage inflammation in the different stages of wound healing.

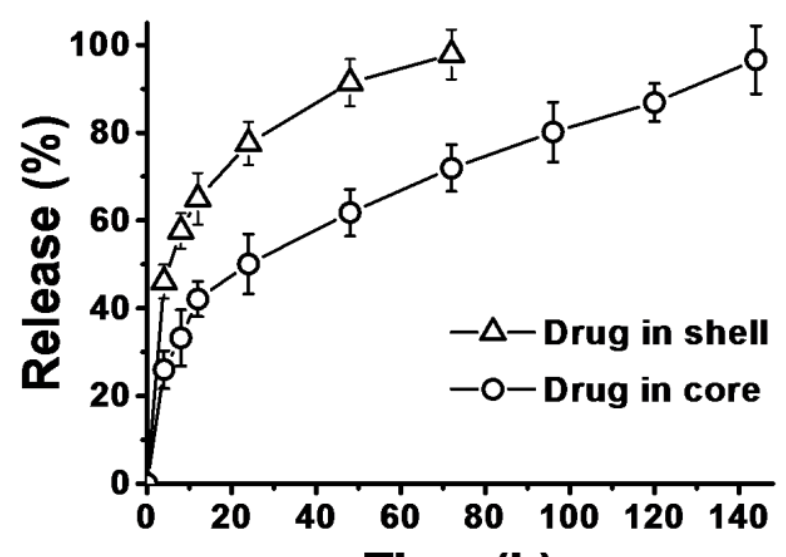

Time (h)

Fig. 4. Drugs release profiles from the core fiber and shell fiber of the fibrous membrane of $7: 3$.

\subsection{Hierarchical micro-nanostructured superhydrophobic surface}

The superhydrophobic performance of lotus leaves to resist wetting of water droplets has been intensively studied. Known as the lotus-leaf effect, the structural basis for superhydrophobicity is attributed to both chemical composition and topological factors.[49, 50] Based on this understanding, bio-inspired surfaces with similar hierarchical micro-nanostructures have shown similar superhydrophobic properties.[49, 50] Since the wetting phenomenon is also important in many biological processes, such as cell adhesion, wound dressings with superhydrophobic surfaces might reduce invasion of bacteria by preventing their adhesion.[51] Creation of hierarchically 
structured surfaces can be readily achieved by electrospinning by decorating micro- and nanobeads onto the surfaces of electrospun fibrous membranes, which has been proven to modify surface wettability of the resulting composite membrane.[27, 52, 53] Accordingly, in our study herein, pure PLA beads have been exploited to enhance surface hydrophobicity.

As expected, after coating with PLA beads, SEM images of the fibrous membranes showed a clear morphological change from a pristine fibrous structure (Fig. 5a) to a mixed structure composed of both fibers and microbeads (Fig. 5b-5f). Under these conditions, the average diameter of the PLA beads was approximately $6 \mu \mathrm{m}$, with good uniformity. Furthermore, the density of PLA beads on the surface could be adjusted by changing the amount of PLA solution sprayed onto a given area of the membrane sample. As the amount of PLA solution increased from 0 to $500 \mu \mathrm{L}$ (per $15 \mathrm{~cm}^{2}$ membrane), the number of PLA beads increased linearly, indicating good adhesion between the fibers and beads during electrospraying. An increased density of PLA microbeads led to increased surface roughness and thus the formation of hierarchical micro-nanostructures, as reflected by their corresponding profilometry images (Fig. S4).
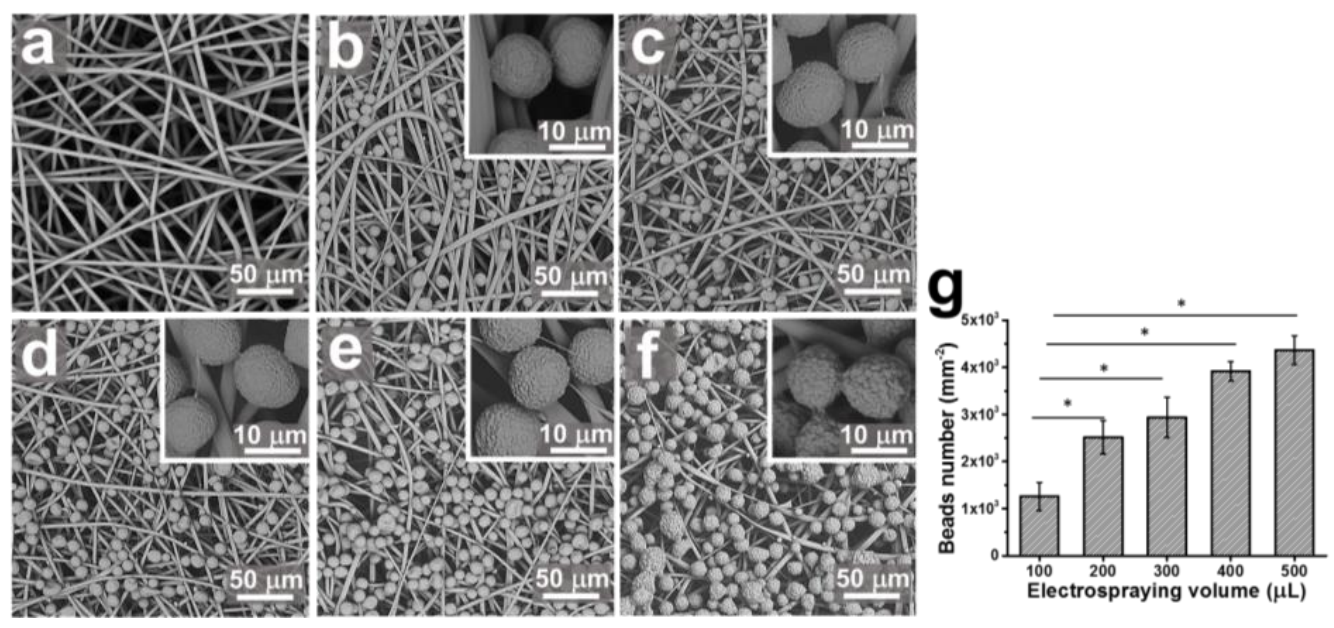
Fig. 5. SEM images of surfaces for untreated membrane (a), $100 \mu \mathrm{L}$ (b), $200 \mu \mathrm{L}$ (c), $300 \mu \mathrm{L}$ (d), $400 \mu \mathrm{L}$ (e) and $500 \mu \mathrm{L}$ electrospraying volume of PLA solution (f) and PLA beads number of different samples $(\mathrm{g})$.

The introduction of hydrophobic PLA microbeads and the creation of rough micronanostructured surfaces generated composite membranes with enhanced surface hydrophobicity.[54, 55] Water contact angle (WCA) values were measured on membranes with different amounts of PLA beads (Fig. 6 and Video S1-S5). As summarized in Fig. 6a, the static WCA values increased with the density of PLA beads (controlled by the volume of PLA solution used in electrospraying). Due to the PLA:PVP blending composition in the shell layer, the surface of pristine membranes was relatively hydrophilic, as indicated by a low WCA value. For samples with the highest PLA density studied here (500 $\mu$ L PLA solution), the WCA value significantly increased to $155 \pm 4.5^{\circ}$, fulfilling the definition of a superhydrophobic surface. The gradual transition from a hydrophilic surface to a superhydrophobic surface (Fig. 6a) was caused by both the increased surface roughness and the chemical composition change after deposition of PLA microbeads.[55]
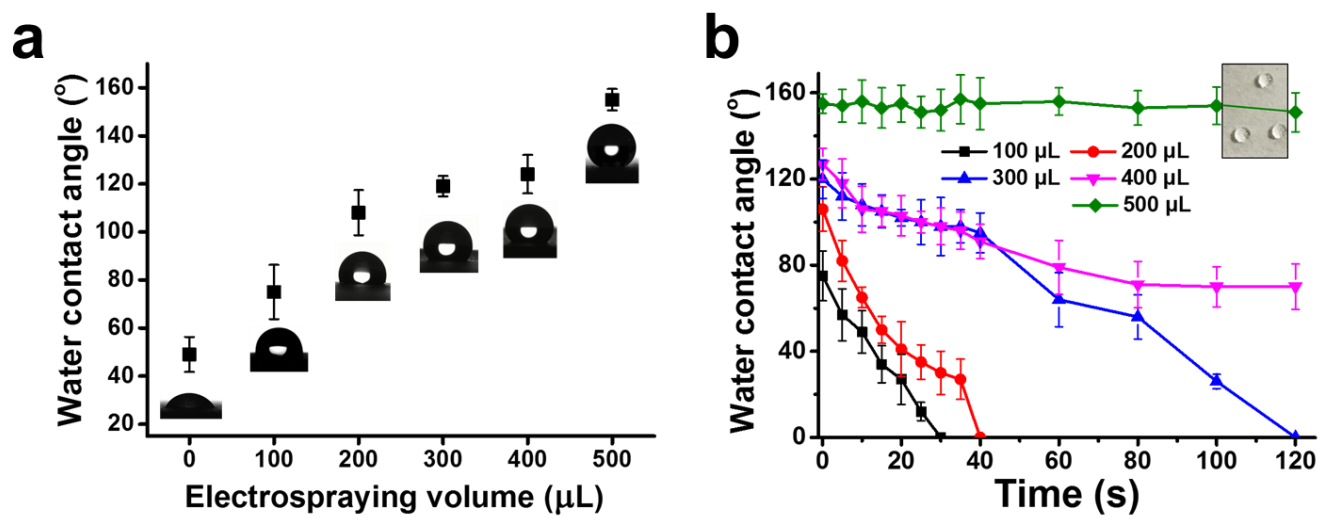

Fig. 6. WCA of the various modified with different concentrations of PLA beads (a). Hydrophobic of various modified with different concentrations of PLA beads at different time periods (the insets is droplets of water on the surface of the $500 \mu \mathrm{L}$ electrospraying volume of PLA solution) (b). 
The dynamic spreading behavior of a water droplet on the composite fibrous membranes was studied. As shown in Fig. 6b and Video S1-S5, water droplets (typically about $2 \mu \mathrm{L}$ in volume) could wet pristine fibrous membranes (without PLA beads) quickly, as suggested by a rapid decrease in WCA. In comparison, the WCA value decrease was much slower for membrane samples coated with PLA beads (Fig. $6 b)$. For the composite membrane modified with $500 \mu \mathrm{L}$ PLA microbeads, water droplets were barely able to spread on the surface for at least $120 \mathrm{~s}$, which also indicated an enhanced surface hydrophobicity and thus enhanced capability to resist bacterial adhesion.[56]

\subsection{In vitro antibacterial performance}

It has been demonstrated that interactions between external bacteria and other microbes with the wound dressing are of critical importance in the early wound healing process.[57, 58] To serve as multifunctional dressing materials, the designed composite membranes should be able to resist bacterial infections. In vitro antibacterial performance of our composite membranes was tested against two common bacteria (gram-positive and gram-negative) of $S$. aureus and E. coli. After seeding the bacteria and cultured on different membrane samples (pristine membranes, composite membranes with PLA microbeads, fibrous membranes with drug payloads) for a specific time, the Live/Dead analysis was applied to determine the bacteria viability. As shown in Fig. 7, under fluorescent microscopy, the green-labeled bacteria were considered alive with intact cell walls, while the red ones were dead with damaged cell 
walls. For both E. coli (Fig. 7a) and S. aureus (Fig. 7b), most bacteria cultured on pristine fibrous membranes were alive. For composite membranes, however, although most bacteria on the membranes were also alive, the density of adhered bacteria was much lower than that on pristine membranes. On the contrary, when both bacteria were treated with fibrous membranes loaded with drugs, nearly all of the adhered bacteria appeared red, indicating poor viability. Results from the Live/Dead assay were also confirmed by SEM images of bacteria on different membranes (Fig. 8). Under SEM observation, live bacteria typically showed certain characteristic shape with uniformity, while dead bacteria with damaged walls were rather irregular in shape. Indeed, the morphologies of $S$. aureus and E. coli on pristine fibrous membranes and composite membranes with PLA microbeads were rod-shaped and rounded, respectively, while bacteria treated with fibrous membranes with drugs showed wrinkled and damaged cells walls, suggesting that these bacteria lost their viability.[59] This is attributed to the rapid release of AMPs loaded in the shell layer of the fibers of the membranes, which led to fast killing of surrounding bacteria (Fig. S5).

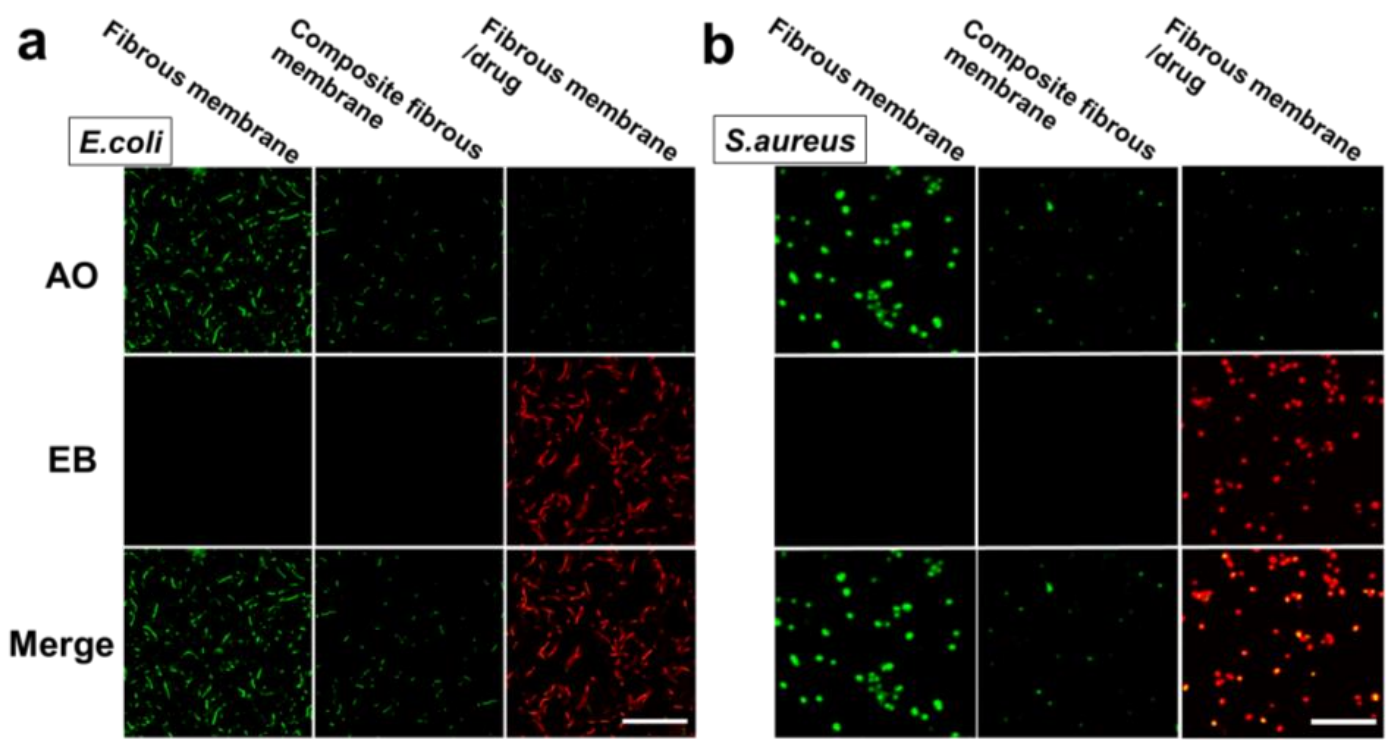


Fig. 7. Laser confocal scanning microscopy images of E. coli cells and $S$. aureus with live/dead assay (The green was AO, indicated bacteria was alive; the red was EB, indicated bacteria was dead). The scale bar is $100 \mu \mathrm{m}$



Fig. 8. SEM images of E. coli cells and $S$. aureus treated with fibrous membranes, composite fibrous membranes and fibrous membranes/drugs (a-f). The antimicrobial activity of the samples (g).

The anti-adhesion effect due to the superhydrophobic nature of the micronanostructured surface with PLA microbeads was confirmed by the significantly reduced bacteria population, as shown in Fig. $8 \mathrm{~b}$ and $8 \mathrm{e}$. These results were reached consensus with previous studies,[50] which confirmed the effectiveness in inhibiting bacteria adhesion and colonization by introducing surface superhydrophobicity. Our results also indicate that the composite membrane with both PLA microbeads and drug loadings can both prevent bacteria invasion and kill surrounding bacteria. Overall, the bio-inspired superhydrophobic property in combination with good antibacterial activity render our composite membrane system a promising candidate in further development of multifunctional wound dressings.

\subsection{In vivo evaluation as wound dressing}


To evaluate the effects of our membrane dressing on the closing of burn skin wound in vivo, the membranes were tested with a full-thickness skin burn model in rat dorsum. Four different dressings, namely, sterile silicone, fibrous membrane and composite fibrous membranes with and without drugs, were applied to the wounds by standard protocols. The subsequent skin repair processes of the treated skin wounds were tracked over a 14-day period. Fig. 9a shows the visual observations of burn wounds treated by different dressings at different post-operation time points. Wounds treated by the composite fibrous membrane loaded with drugs showed a significantly higher wound healing rate than all other groups tested. At day 10, the remaining wound areas under the composite membrane dressing with drugs were also significantly smaller than other groups. At day 14, wounds treated by our composite fibrous membrane with drugs appeared to be fully recovered. Therefore, comparing to conventional wound dressing materials and other control groups, our composite fibrous membranes that combine a superhydrophobic surface with loaded core/shell fibers show the capability in promoting the wound healing process.

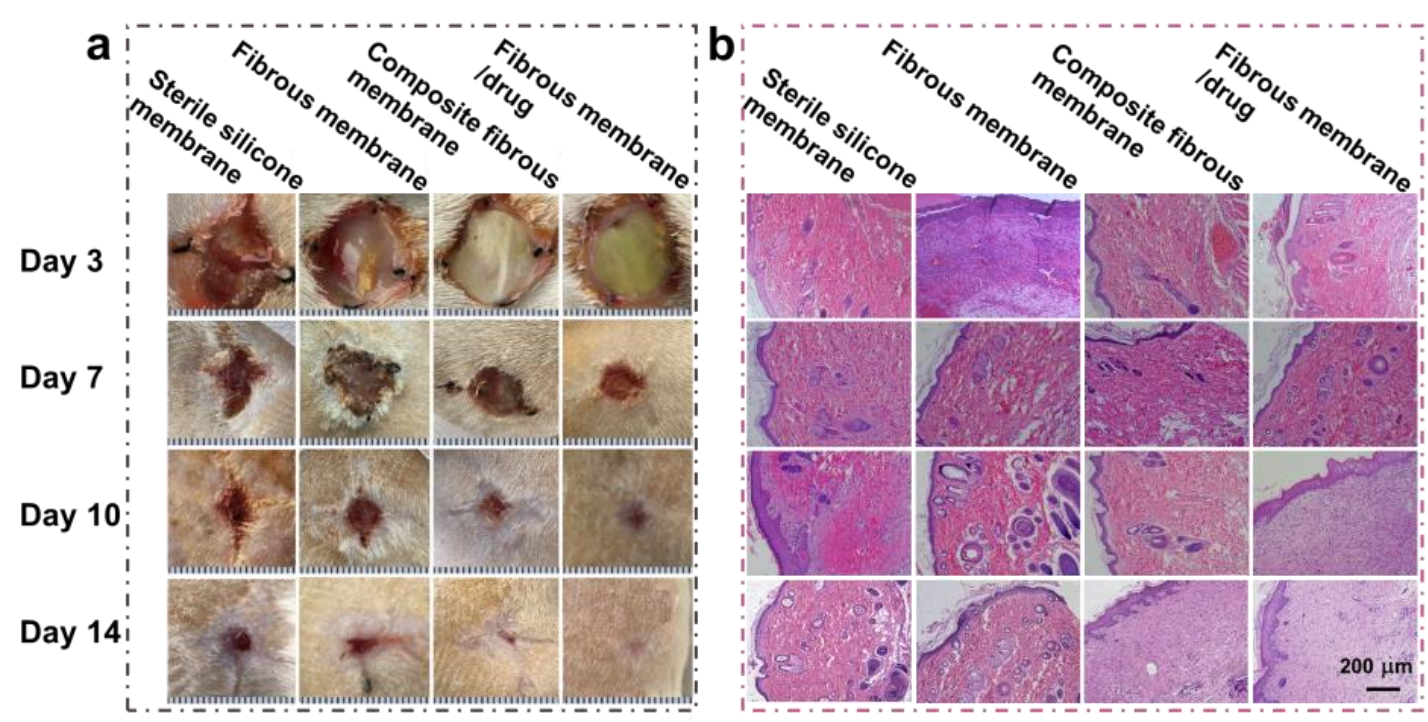


Fig. 9. Effect of the membranes on wound healing. (a) Appearances of the size change of the large excision wounds made in full-thickness skin with different time periods. (b) Histological staining with hematoxylin and eosin of the wound sites treated with the membranes.

Wound healing is a fundamental natural reaction to tissue damage. The healing process and skin regeneration are relevant to inflammation, epithelialization, forming of granulation tissue and tissue remodeling. To determine the effects on our composite membrane dressing on these different processes involved in wound healing, the wounded skin sections were treated with $\mathrm{H} \& \mathrm{E}$ staining for histomorphological observations of the regenerated tissues (Fig. 9b) and MT staining to investigate the degree of collagen deposition during the healing process (Fig. 10a). Plenty of fibroblasts could be observed in each group in the early stages following operation. Compared with the sterile silicone control, all types of fibrous membrane gave extended re-epithelization of desquamated epithelial areas and less inflammatory cell infiltrations in granulation tissues. Along with the progress of tissue repair, the composite fibrous membrane with drugs displayed a decreased cell number and abundant collagen, which were subsequently filled with dense collagen fibers and differentiated skin appendages. Correspondingly, the amount of collagen in granulation tissues were increased in our drug-loaded composite fibrous membrane. On the contrary, other fibrous membranes without drugs and the sterile silicone control postponed these histopathological changes that were related to the burn wound healing process. 


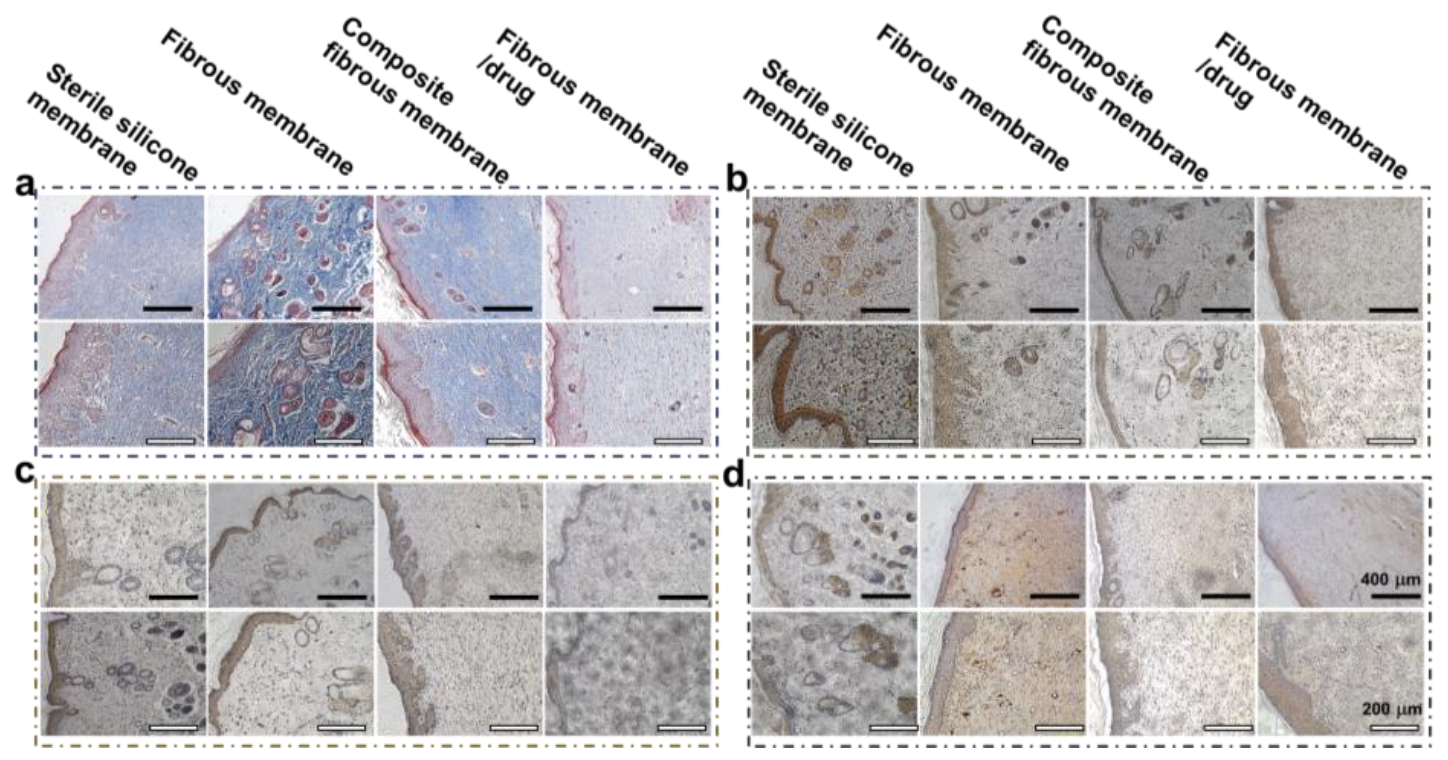

Fig. 10. Wound sections on days 14 were stained with (a) Masson's Trichrome and the expression of (b) IL-1 $\beta$, (c) IL-6 $\beta$ and (d) TNF- $\alpha$. Representative light microscopy images of sections are presented for all four groups.

Proinflammatory cytokines are primal cytokine mediators of the acute inflammatory response to tissue damage, of which the release of somatostatin and inhibit the release of growth hormones could be stimulated by TNF- $\alpha$, IL- 6 and IL- $1 \beta$. The major function of growth hormone is to promote protein synthesis and thus tissue repair, while the restrained release of growth hormone would postpone wound healing. We determined the degrees of expression of the three proinflammatory cytokines in sectioned skin samples. The results (Fig. 10b-10d) showed decreased levels of these proinflammatory cytokines in the experimental group treated with drug-loaded composite membrane dressings, which was favorable to wound healing and could explain the observed accelerated wound healing using our novel drug-loaded superhydrophobic fiber/bead dressing materials.

\section{Conclusions}


In summary, we report the preparation and characterization of a composite membrane composed of PLA:PVP/PLA:PEG core/shell microfibers, which was further decorated with PLA microbeads and loaded with two model drugs (partitioned in the core and shell, respectively) via electrospinning. The optimal blending ratio of PLA and PVP in the shell layer was screened to show that 7:3 (w/w), which could lead to greater thermal stability, mechanical properties and swelling capability. In vitro drug release studies confirmed a tailored release profile with programmable features. AMPs loaded in the shell layer showed a faster release to deal with acute bacterial infection, while curcumin loaded in the core displayed a postponed release suitable to manage inflammation in the later stages of wound healing. The electrospun fibrous membranes were further modified with PLA microbeads via electrospraying to bring in superhydrophobic properties, which could contribute to the prevention of bacterial adhesion. The resulting composite fibrous membranes with drug loadings demonstrated notable in vitro antibacterial activity against both gram-positive and gram-negative, as proved by the Live/Dead assay and SEM observations. In vivo evaluation of this multifunctional dressing material was performed in a rat model. Accelerated wound healing with reduced wound sizes and repair time was observed, attributed to the successful management of bacterial invasion and inflammation. Overall, we believe that this work provides an innovative approach to address burn wound healing using rationally designed, multifunctional dressing materials based on decorated fiber/bead fibers. The combined antibacterial activity and profound wound healing effects make our drug- 
loaded composite fibrous membranes an advanced system for developing effective burn treatments of the future.

\section{Conflict of interest}

The authors declare no competing financial interest.

\section{Supporting Information}

Fourier transform infrared (FT-IR) spectroscopy, swelling ratio and thermal degradation behaviors of the fibrous membranes. Antibacterial activity of the micronanostructured membranes, and images of $E$. coli cultured on a series of composite fibrous membranes with different PLA beads contents. This material is available free of charge.

\section{Acknowledgments}

The authors thank the financial support from the National Key R\&D Program of China (No.2017YFC11050003), Guangdong Innovative and Entrepreneurial Research Team Program (No. 2016ZT06C322), the Fundamental Research Funds for the Central Universities (No. 2018PY05). PDT thanks the State Administration for Foreign Experts Affairs (SAFEA) and the Royal Society of Chemistry for a Visiting Researcher Program grant to China.

\section{References}

[1] A. J. Singer, L. Berruti, H. C. Thode, S. A. Mcclain, Standardized burn model using a multiparametric histologic analysis of burn depth. Acad Emerg Med. 7 (2000) $1-6$.

[2] P. A. Brigham, E. Mcloughlin, Burn incidence and medical care use in the United States: estimates, trends, and data sources. J Burn Care Rehabil. 17 (1996) 95-107. 
[3] M. Loss, V. Wedler, W. Künzi, C. Meuli-Simmen, V. Meyer, Artificial skin, splitthickness autograft and cultured autologous keratinocytes combined to treat a severe burn injury of 93\% of TBSA. Burns 26 (2000) 644-652.

[4] M. Takeo, W. Lee, M. Ito, Wound healing and skin regeneration. CSH perspect med. 5 (2015) a023267.

[5] M. Norouzi, I. Shabani, F. Atyabi, M. Soleimani, EGF-loaded nanofibrous scaffold for skin tissue engineering applications. Fiber Polym. 16 (2015) 782-787.

[6] S. Böttcher-Haberzeth, T. Biedermann, E. Reichmann, Tissue engineering of skin. Burns 36 (2010) 450-460.

[7] D. Li, G. Jiao, W. Zhang, X. Chen, R. Ning, C. Du, Hybrid scaffolding strategy for dermal tissue reconstruction: a bioactive glass/chitosan/silk fibroin composite. RSC Advances. 6 (2016) 19887-19896.

[8] G. Arturson, Pathophysiology of the burn wound and pharmacological treatment. The Rudi Hermans Lecture, 1995. Burns 22 (1996) 255-274.

[9] G. C. Gurtner, S. Werner, Y. Barrandon, M. T. Longaker, Wound repair and regeneration. Nature 453 (2008) 314-321.

[10] M. Abrigo, S. L. McArthur, P. Kingshott, Electrospun nanofibers as dressings for chronic wound care: advances, challenges, and future prospects. Macromol Biosci. 14 (2014) 772-792.

[11] H. Hajiali, M. Summa, D. Russo, A. Armirotti, V. Brunetti, R. Bertorelli, A. Athanassiou, E. Mele, Alginate-lavender nanofibers with antibacterial and antiinflammatory activity to effectively promote burn healing. J Mater Chem B. 4 (2016) 1686-1695.

[12] S. B. Mahjour, X. Fu, X. Yang, J. Fong, F. Sefat, H. Wang, Rapid creation of skin substitutes from human skin cells and biomimetic nanofibers for acute fullthickness wound repair. Burns 41 (2015) 1764-1774.

[13] E. K. Yim, K. W. Leong, Significance of synthetic nanostructures in dictating cellular response. Nanomedicine: Nanotechnology, Nanomed Nanotechnol. 1 (2005) 10-21.

[14] R. Huang, W. Li, X. Lv, Z. Lei, Y. Bian, H. Deng, H. Wang, J. Li, X. Li, Biomimetic LBL structured nanofibrous matrices assembled by chitosan/collagen for promoting wound healing. Biomaterials 53 (2015) 58-75.

[15] Y.L. Liu, T.W. Chuo, Self-healing polymers based on thermally reversible DielsAlder chemistry. Polym Chem. 4 (2013) 2194-2205.

[16] R. P. Wool, Self-healing materials: a review. Soft Matter. 4 (2008) 400-418.

[17] X.Y. Li, Y.C. Li, D.G. Yu, Y.-Z. Liao, X. Wang, Fast disintegrating quercetinloaded drug delivery systems fabricated using coaxial electrospinning. Int J Mol Sci. 14 (2013) 21647-21659.

[18] X. Lu, C. Wang, Y. Wei, One-Dimensional Composite Nanomaterials: Synthesis by Electrospinning and Their Applications. Small 5 (2009) 2349-2370.

[19] M. Zamani, M. P. Prabhakaran, S. Ramakrishna, Advances in drug delivery via electrospun and electrosprayed nanomaterials. Int J Nanomed. 8 (2013) 2997-3017. 
[20] Y. Lu, S. Sathasivam, J. Song, C. R. Crick, C. J. Carmalt, I. P. Parkin, Robust self-cleaning surfaces that function when exposed to either air or oil. Science 347 (2015) 1132-1135.

[21] L. Chen, X. Liu, B. Su, J. Li, L. Jiang, D. Han, S. Wang, Aptamer-mediated efficient capture and release of T lymphocytes on nanostructured surfaces. Adv Mater. 23 (2011) 4376-4380.

[22] T. Lv, Z. Cheng, D. Zhang, E. Zhang, Q. Zhao, Y. Liu, L. Jiang, Superhydrophobic Surface With Shape Memory Micro/Nanostructure and Its Application in Rewritable Chip for Droplet Storage. ACS Nano. 10 (2016) 93799786.

[23] G. Wei, Z. Wang, X. Zhao, J. Feng, S. Wang, J. Zhang, C. An, A facile approach to fabricate superhydrophobic and corrosion resistant surface. MaterRes Express. 2 (2014) 015501.

[24] L. Jiang, Y. Zhao, J. Zhai, A lotus-leaf-like superhydrophobic surface: a porous microsphere/nanofiber composite film prepared by electrohydrodynamics. Angew ChemInt Edit. 116 (2004) 4438-4441.

[25] P. Guo, Y. Zheng, M. Wen, C. Song, Y. Lin, L. Jiang, Icephobic/Anti-Icing Properties of Micro/Nanostructured Surfaces. Adv Mater. 24 (2012) 2642-2648. [26] H. Attia, D. J. Johnson, C. J. Wright, N. Hilal, Robust superhydrophobic electrospun membrane fabricated by combination of electrospinning and electrospraying techniques for air gap membrane distillation. Desalination 446 (2018) 70-82.

[27] J. Wu, X. Li, Y. Wu, G. Liao, P. Johnston, P. D. Topham, L. Wang, Rinseresistant superhydrophobic block copolymer fabrics by electrospinning, electrospraying and thermally-induced self-assembly. Appl Surf Sci. 422 (2017) 769777.

[28] L. Wang, P. D. Topham, O. O. Mykhaylyk, H. Yu, A. J. Ryan, J. P. A. Fairclough, W. Bras, Self-Assembly-Driven Electrospinning: The Transition from Fibers to Intact Beaded Morphologies. Macromol Rapid Commun. 36 (2015) 14371443.

[29] M. Chromek, Z. Slamová, P. Bergman, L. Kovács, L. u. Podracká, I. Ehrén, T. Hökfelt, G. H. Gudmundsson, R. L. Gallo, B. Agerberth, The antimicrobial peptide cathelicidin protects the urinary tract against invasive bacterial infection. Nat Med. 12 (2006) 636-641.

[30] F. H. Login, S. Balmand, A. Vallier, C. Vincent-Monégat, A. Vigneron, M.

Weiss-Gayet, D. Rochat, A. Heddi, Antimicrobial peptides keep insect endosymbionts under control. Science 334 (2011) 362-365.

[31] S. Julie, M. Jurenka, Anti-inflammatory properties of curcumin, a major constituent. Altern Med Rev. 14 (2009).

[32] X. Zhao, Z. Yuan, L. Yildirimer, J. Zhao, Z. Y. W. Lin, Z. Cao, G. Pan, W. Cui, Tumor-Triggered Controlled Drug Release from Electrospun Fibers Using Inorganic Caps for Inhibiting Cancer Relapse. Small 11 (2015) 4284-4291. 
[33] L. Wang, J. Chen, L. Shi, Z. Shi, L. Ren, Y. Wang, The promotion of antimicrobial activity on silicon substrates using a "click" immobilized short peptide. Chem Commun. 50 (2014) 975-977.

[34] L. Mei, Z. Lu, W. Zhang, Z. Wu, X. Zhang, Y. Wang, Y. Luo, C. Li, Y. Jia, Bioconjugated nanoparticles for attachment and penetration into pathogenic bacteria. Biomaterials 34 (2013) 10328-10337.

[35] M. Zamani, M. P. Prabhakaran, S. Ramakrishna, Advances in drug delivery via electrospun and electrosprayed nanomaterials. Int J nanomed. 8 (2013) 2997.

[36] N. Kaerkitcha, S. Chuangchote, T. Sagawa, Control of physical properties of carbon nanofibers obtained from coaxial electrospinning of PMMA and PAN with adjustable inner/outer nozzle-ends. Nanoscale res lett. 11 (2016) 186.

[37] S. Zereshki, A. Figoli, S. Madaeni, S. Simone, J. Jansen, M. Esmailinezhad, E. Drioli, Poly (lactic acid)/poly (vinyl pyrrolidone) blend membranes: Effect of membrane composition on pervaporation separation of ethanol/cyclohexane mixture. J Membrane Sci. 362 (2010) 105-112.

[38] S. Zereshki, A. Figoli, S. Madaeni, S. Simone, E. Drioli, Pervaporation separation of methanol/methyl tert-butyl ether with poly (lactic acid) membranes. J Appl Polym Sci. 118 (2010) 1364-1371.

[39] M. Kumar, R. Rahikainen, D. Unruh, V. P. Hytonen, C. Delbruck, R. Sindelar, F. Renz, Mixture of PLA-PEG and biotinylated albumin enables immobilization of avidins on electrospun fibers. J Biomed Mater Res A. 2016.

[40] P. Pihui, M. Wei, G. Fei, D. Yulin, Superhydrophobic film fabricated by controlled microphase separation of PEO-PLA mixture and its transparence property. Appl Surf Sci. 273 (2013) 184-191.

[41] H.Z. Li, S.C. Chen, Y.Z. Wang, Thermoplastic PVA/PLA blends with improved processability and hydrophobicity. Ind Eng Chem Res. 53 (2014) 17355-17361. [42] N. Tuancharoensri, G. M. Ross, S. Mahasaranon, P. D. Topham, S. Ross, Ternary blend nanofibres of poly (lactic acid), polycaprolactone and cellulose acetate butyrate for skin tissue scaffolds: influence of blend ratio and polycaprolactone molecular mass on miscibility, morphology, crystallinity and thermal properties. Polym Int. 66 (2017) 1463-1472.

[43] Q. Yao, J. G. Cosme, T. Xu, J. M. Miszuk, P. H. Picciani, H. Fong, H. Sun, Three dimensional electrospun PCL/PLA blend nanofibrous scaffolds with significantly improved stem cells osteogenic differentiation and cranial bone formation. Biomaterials 115 (2017)115-127.

[44] E. Zhmayev, D. Cho, Y. L. Joo, Nanofibers from gas-assisted polymer melt electrospinning. Polymer 51 (2010) 4140-4144.

[45] H. Li, M. A. Huneault, Effect of nucleation and plasticization on the crystallization of poly (lactic acid). Polymer 48 (2007) 6855-6866.

[46] A. Moghe, B. Gupta, Co-axial electrospinning for nanofiber structures: preparation and applications. Polym Rev. 48 (2008) 353-377.

[47] E. Redolfi Riva, A. Desii, S. Sartini, C. La Motta, B. Mazzolai, V. Mattoli, PMMA/polysaccharides nanofilm loaded with adenosine deaminase inhibitor for targeted anti-inflammatory drug delivery. Langmuir 29 (2013) 13190-13197. 
[48] H. Lu, Q. Wang, G. Li, Y. Qiu, Q. Wei, Electrospun water-stable zein/ethyl cellulose composite nanofiber and its drug release properties. Mater Sci Eng. 74 (2017) 86-93.

[49] A. Razmjou, E. Arifin, G. Dong, J. Mansouri, V. Chen, Superhydrophobic modification of $\mathrm{TiO}_{2}$ nanocomposite PVDF membranes for applications in membrane distillation. J Membrane Sci. 415 (2012) 850-863.

[50] S. H. Yoon, N. Rungraeng, W. Song, S. Jun, Superhydrophobic and superhydrophilic nanocomposite coatings for preventing Escherichia coli K-12 adhesion on food contact surface. J Food Eng. 131 (2014) 135-141.

[51] P. Tang, W. Zhang, Y. Wang, B. Zhang, H. Wang, C. Lin, L. Zhang, Effect of superhydrophobic surface of titanium on staphylococcus aureus adhesion. $\mathrm{J}$

Nanomater. (2011) 3585-3585.

[52] X. Wang, J. Wang, Y. Si, B. Ding, J. Yu, G. Sun, W. Luo, G. Zheng, Nanofibernet-binary structured membranes for highly sensitive detection of trace $\mathrm{HCl}$ gas.

Nanoscale 4 (2012) 7585-7592.

[53] X. Tang, Y. Si, J. Ge, B. Ding, L. Liu, G. Zheng, W. Luo, J. Yu, In situ polymerized superhydrophobic and superoleophilic nanofibrous membranes for gravity driven oil-water separation. Nanoscale 5 (2013) 11657-11664.

[54] P. Zhang, S. Wang, S. Wang, L. Jiang, Superwetting surfaces under different media: Effects of surface topography on wettability. Small 11 (2015) 1939-1946.

[55] S. Wang, Y. Zhu, F. Xia, J. Xi, N. Wang, L. Feng, L. Jiang, The preparation of a superhydrophilic carbon film from a superhydrophobic lotus leaf. Carbon 44 (2006) 1848-1850.

[56] H. Yang, Y. Deng, Preparation and physical properties of superhydrophobic papers. J Colloid Interf Sci. 325 (2008) 588-593.

[57] W.T. Lin, H.L. Tan, Z.L. Duan, B. Yue, R. Ma, G. He, T.-t. Tang, Inhibited bacterial biofilm formation and improved osteogenic activity on gentamicin-loaded titania nanotubes with various diameters. Int J Nanomed.9 (2014) 1215.

[58] H. Qin, H. Cao, Y. Zhao, C. Zhu, T. Cheng, Q. Wang, X. Peng, M. Cheng, J. Wang, G. Jin, In vitro and in vivo anti-biofilm effects of silver nanoparticles immobilized on titanium. Biomaterials 35 (2014) 9114-9125.

[59] K. Matsuzaki, Control of cell selectivity of antimicrobial peptides. BBA Biomembranes 1788 (2009) 1687-1692. 